\title{
Edge State Transport of Separately Contacted Bilayer Systems in the Fractional Quantum Hall Regime
}

\author{
Daijiro Yoshioka and Kentaro Nomura \\ Department of Basic Science, The University of Tokyo \\ 3-8-1 Komaba, Meguro, Tokyo 153-8902, Japan
}

\begin{abstract}
Hall and diagonal resistances of bilayer fractional quantum Hall systems are discussed theoretically. The bilayers have electrodes attached separately to each layer. They are assumed to be coupled weakly by interlayer tunneling, while the interlayer Coulomb interaction is negligibly small. It is shown that source-drain voltage dependence of the resistances reflects the Luttinger liquid parameter of the edge state.
\end{abstract}

73.40.-c, 73.40.Gk,73.40.Hm, FQHE, bilayer, tunneling, Luttinger liquid

Recently it has become possible to fabricate bilayer systems where electrodes are attached separately to each layers [1]. Most experimental and theoretical investigations have been done for the phenomenon called as Coulomb drag in these systems, which occurs only when the system is in the compressible phase. However, even in the incompressible states non-trivial transport phenomena are expected to occur if the two-layers are coupled through tunneling. Actually, in the integer quantum Hall regime deviation from the quantized Hall value and finite dissipation have been observed experimentally [2], and analyzed theoretically [3, 1 . The purpose of the present paper is to investigate the consequences of the tunneling for weakly coupled bilayer systems in the fractional quantum Hall regime.

For the bilayer system, where current is supplied to only one of the layer, the two layers are out of equilibrium, especially at the edges. The tunneling cause the two layers to approach to the equilibrium. It is known that the tunneling in the bulk is suppressed as long as the voltage difference between the two-layers is small [5]. Therefore we assume that the tunneling occurs only between the chiral Luttinger liquids realized at the sample edge.

However, even at the edges, it has been shown that for the principal quantum Hall states realized at filling factor $1 / q(q>1)$ the tunneling is irrelevant and suppressed in contrast to the integer quantum Hall (IQH) case, where it is relevant [6]. For the case of FQH, The irrelevancy of the tunneling gives power law dependence of the tunneling current on the voltage difference or on the temperature [7]. The power is given by the Luttinger liquid parameter, which is nothing but the filling factor of the system. This theory suggests new experiment to investigate the Luttinger liquid property of the fractional quantum Hall edges.

Following [3] we introduce local effective chemical potential $\mu_{\sigma}(x)$ to describe the current flowing in the $\sigma$ layer at coordinate $x$ along the edge, where $\sigma= \pm$ is the layer index. The introduction of the effective chemical potential does not necessarily mean that each layer is in thermal equilibrium [8]. The current in each layer is given by

$$
I_{\sigma}(x)=\frac{\nu e}{h}\left[\mu_{\sigma}(x)-\epsilon_{0}\right]
$$

where $\epsilon_{0}$ is a common origin to measure energy and defines the edge current. Due to the chirality of the edges the sum of the current $I_{+}(x)+I_{-}(x)$ is conserved along the edges both from source to drain and from drain to source. However, the tunneling current causes the change in the current in each layers. Since the electrons flow at a constant velocity along the edges, the temporal evolution of the difference in the chemical potential is projected to the spatial evolution of the chemical potential. The spatial evolution should be described by Boltzman type differential equation as follows

$$
\frac{\mathrm{d} \mu_{\sigma}(x)}{\mathrm{d} x}=-\frac{1}{\xi}\left[\mu_{\sigma}(x)-\mu_{-\sigma}(x)\right]^{\lambda} .
$$

In this equation $\lambda$ is given by the Luttinger parameter of the edge state. For the standard theory of the edge state in the principal FQH state at $\nu=1 / q, \lambda=2 q-1$ [9]. However, we can consider $\lambda$ as a parameter to be determined experimentally. The parameter $\xi$, which has the meaning of the relaxation length for the $\mathrm{IQH}$, is inversely proportional to the tunneling probability. We solve this equation under the condition that the total current through the sample is $I$, and only the minus layer is connected to the source and drain electrodes. We assume that the current is injected ideally without reflection into the upper edge of the minus layer from the source at $x=0$, and drained at $x=L$, namely $L$ is the sample length. Thus the boundary condition for the minus layer is $\mu_{-}(0+)=\mu_{\mathrm{S}}$, and $\mu_{-}(L-)=\mu_{\mathrm{D}}+(h / \nu e) I$. Similarly boundary condition for the lower edge of the minus layer is $\mu_{-}(L+)=\mu_{\mathrm{D}}$, and $\mu_{-}(2 L-)=\mu_{\mathrm{S}}-(h / \nu e) I$, where neglecting the width of the electrodes, the edge state extends from the drain at $x=L$ to the source at $x=2 L \equiv 0$. On the other hand, since the upper layer is not connected to the current electrodes, the condition for $\mu_{+}(x)$ is $\mu_{+}(0-)=\mu_{+}(0+)$ and $\mu_{+}(L-)=\mu_{+}(L+)$.

To solve the equations we introduce an auxiliary variable $\zeta$ such that 


$$
\begin{aligned}
\left(\mu_{\mathrm{S}}-\mu_{\mathrm{D}}\right) \zeta & =\mu_{-}(0+)-\mu_{-}(L-) \\
& =-\left[\mu_{+}(0)-\mu_{+}(L)\right] \\
& =\mu_{-}(L+)-\mu_{-}(0-) .
\end{aligned}
$$

This $\zeta$ is determined by the following transcendental equation:

$$
\begin{aligned}
(1-3 \zeta)^{1-\lambda} & -(1+\zeta)^{1-\lambda} \\
& =2(\lambda-1) \frac{L}{\xi}\left[\frac{1}{2}\left(\mu_{\mathrm{S}}-\mu_{\mathrm{D}}\right)\right]^{\lambda-1} .
\end{aligned}
$$

Once $\zeta$ is determined, $\mu_{ \pm}(x)$ is given: Namely for $0<$ $x<L$,

$$
\begin{aligned}
\mu_{ \pm}(x)= & \frac{1}{4}\left[(3-\zeta) \mu_{\mathrm{S}}+(1+\zeta) \mu_{\mathrm{D}}\right] \\
\mp & \frac{1}{2}\left[\left\{\frac{1+\zeta}{2}\left(\mu_{\mathrm{S}}-\mu_{\mathrm{D}}\right)\right\}^{1-\lambda}\right. \\
& \left.+\frac{2(\lambda-1)}{\xi} x\right]^{1 /(1-\lambda)},
\end{aligned}
$$

and for $L<x<2 L$,

$$
\begin{aligned}
\mu_{ \pm}(x)= & \frac{1}{4}\left[(1+\zeta) \mu_{\mathrm{S}}+(3-\zeta) \mu_{\mathrm{D}}\right] \\
\pm & \frac{1}{2}\left[\left\{\frac{1+\zeta}{2}\left(\mu_{\mathrm{S}}-\mu_{\mathrm{D}}\right)\right\}^{1-\lambda}\right. \\
& \left.+\frac{2(\lambda-1)}{\xi}(x-L)\right]^{1 /(1-\lambda)} .
\end{aligned}
$$

It is easily verified that $\mu_{ \pm}(x)$ satisfies the differential equation with the proper boundary conditions.

Now, the current through the sample is given as follows:

$$
I=\nu \frac{e}{h}(1-\zeta)\left(\mu_{\mathrm{S}}-\mu_{\mathrm{D}}\right)
$$

Therefore the source-drain conductance is given by

$$
G=\frac{\nu e^{2}}{h}(1-\zeta)
$$

Hall and diagonal resistances when the probe electrodes are attached to the minus layer at $x=a, x=L-a$, and $x=2 L-a$ are calculated from eqs.(5) and (6):

$$
\begin{aligned}
R_{\mathrm{H}} \equiv & \frac{\mu_{-}(a)-\mu_{-}(2 L-a)}{e I} \\
= & \left(\frac{h}{\nu e^{2}}\right)\left\{\frac{1}{2}+\frac{1}{4(1-\zeta)}\left[\frac{L-a}{L}(1+\zeta)^{1-\lambda}\right.\right. \\
& \left.+\frac{a}{L}(1-3 \zeta)^{1-\lambda}\right]^{1 /(1-\lambda)} \\
+ & \frac{1}{4(1-\zeta)}\left[\frac{a}{L}(1+\zeta)^{1-\lambda}\right. \\
& \left.\left.+\frac{L-a}{L}(1-3 \zeta)^{1-\lambda}\right]^{1 /(1-\lambda)}\right\}
\end{aligned}
$$

and

$$
R_{x x} \equiv \frac{\mu_{-}(a)-\mu_{-}(L-a)}{e I}
$$

$$
\begin{aligned}
= & \left(\frac{h}{\nu e^{2}}\right) \frac{1}{4(1-\zeta)}\left\{\left[\frac{L-a}{L}(1+\zeta)^{1-\lambda}\right.\right. \\
& \left.+\frac{a}{L}(1-3 \zeta)^{1-\lambda}\right]^{1 /(1-\lambda)} \\
- & {\left[\frac{a}{L}(1+\zeta)^{1-\lambda}\right.} \\
& \left.\left.+\frac{L-a}{L}(1-3 \zeta)^{1-\lambda}\right]^{1 /(1-\lambda)}\right\} .
\end{aligned}
$$

These results are shown in Figs. 1 and 2. First in Fig.1 the normalized conductance $G /\left(\nu e^{2} / h\right)=(1-\zeta)$ is plotted as a function of $(L / \xi)\left[\left(\mu_{\mathrm{S}}-\mu_{\mathrm{D}}\right) / 2\right]^{\lambda-1}$. As the tunneling current increases either as the length $L$ or $\left(\mu_{\mathrm{S}}-\mu_{\mathrm{D}}\right)$ increase or $\xi$ decreases, the source-drain conductance decreases. This is due to the increase of the back-scattered current through the plus layer. A peculiarity of the FQH case is that it depends on $\left(\mu_{\mathrm{S}}-\mu_{\mathrm{D}}\right)$. The conductance tends to $(2 / 3)\left(\nu e^{2} / h\right)$ as the tunneling current increases.

In Fig.2 the Hall and longitudinal resistances, which are normalized by $h / \nu e^{2}$, are plotted for the choice of $a=0.2 L$. Effect on the longitudinal resistance is smaller in the fractional case. The Hall resistance tends to $1 / 2$ of the isolated layer case in the large tunneling current limit, while $R_{x x}$ tends to zero similarly to the IQH case.

The results in this paper show that if $\left(\mu_{\mathrm{S}}-\mu_{\mathrm{D}}\right)$ dependence of the various quantity is measured for tunnel-coupled bilayer systems, we can deduce the parameter $\lambda$, or the Luttinger parameter of the edge state. Of course such an experiment should be done at low temperature and for small source-drain voltage, since tunneling in the bulk must be negligible. We hope such an experiment will be done in the near future. This work is supported by Grant-in-Aid for Scientific Research (C) 10640301.

[1] J.P. Eisenstein, L.N. Pfeiffer and K.W. West: Appl. Phys. Lett. 57 (1990) 2324.

[2] Y. Ohno, M. Foley and H. Sakaki: Phys. Rev. B54 (1996) R2319.

[3] D. Yoshioka and A.H. MacDonald: Phys. Rev. B53 (1996) R16168.

[4] D. Yoshioka and A.H. MacDonald: Physica E 1 (1997) 167.

[5] J.P. Eisenstein, L.N. Pfeiffer and K.W. West: Phys. Rev. Lett. 69 (1992) 3804.

[6] K. Nomura and D. Yoshioka: to be published in J. Phys. Soc. Jpn., cond-mat/9904192.

[7] M. Grayson, D.C. Tsui, L.N. Pfeiffer, K.W. West and A.M. Chang: Phys. Rev. Lett. 80 (1998) 1062.

[8] If the interlayer relaxation is faster than the intralayer relaxation, each edge will be in thermal equilibrium, and the effective chemical potential is nothing but the true chemical potential.

[9] X.G. Wen: Phys. Rev. B44 (1991) 5708. 


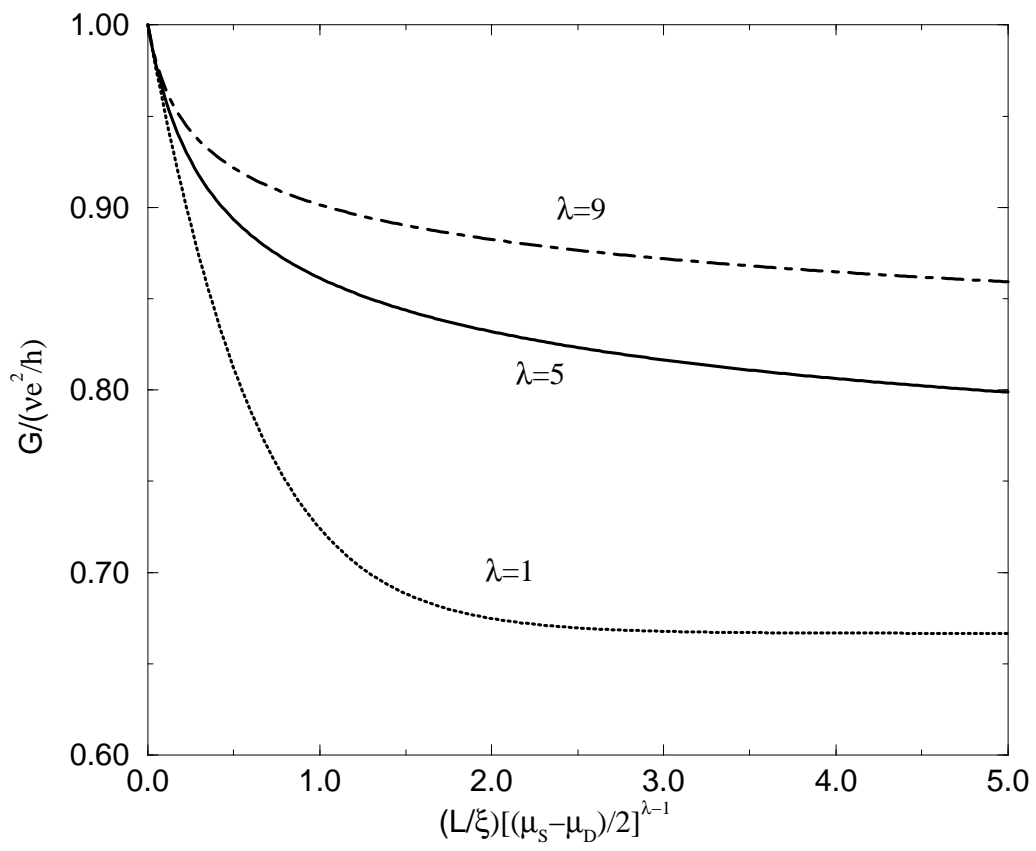

FIG. 1. Two terminal conductance $G$ scaled by $\nu e^{2} / h$ is shown as a function of tunneling strength $(L / \xi)\left[\left(\mu_{\mathrm{S}}-\mu_{\mathrm{D}}\right) / 2\right]^{\lambda-1}$ for $\lambda=1,5$, and 9 by dotted, solid and dot-dashed lines, respectively.

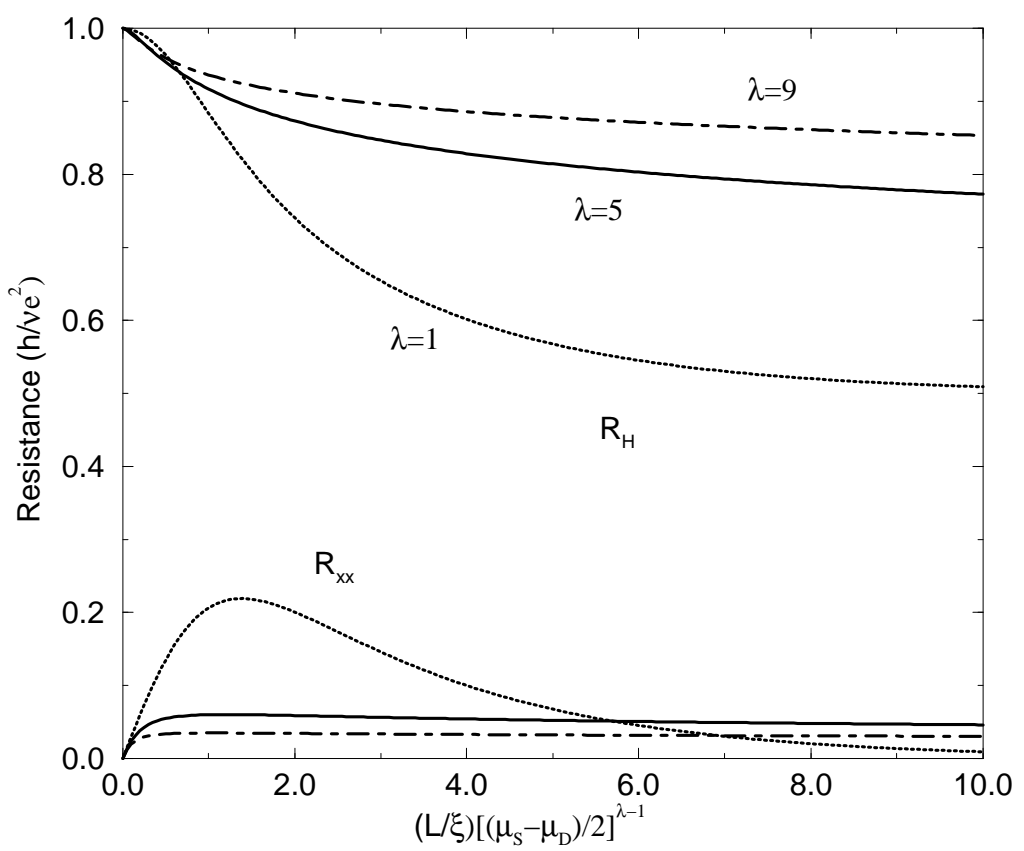

FIG. 2. The diagonal and Hall resistances $R_{x x}$ and $R_{\mathrm{H}}$ scaled by $h / \nu e^{2}$ are shown as a function of tunneling strength $(L / \xi)\left[\left(\mu_{\mathrm{S}}-\mu_{\mathrm{D}}\right) / 2\right]^{\lambda-1}$ for $\lambda=1,5$, and 9 by dotted, solid and dot-dashed lines, respectively. The length of the sample is $L$ and voltage probes are attached at $0.2 L$ from the source and drain. 